\title{
Subdural Hemorrhage Related to Vitamin K Deficiency in an Infant with Choledochal Cyst
}

Sohyung Park ${ }^{1}$, Jang Gyu Cha ${ }^{2}$, Byung-Ha Choi ${ }^{1}$

${ }^{1}$ Department of Forensic Medicine, National Forensic Service, Wonju, Korea, ${ }^{2}$ Department of Radiology, Soonchunhyang University Bucheon Hospital, Bucheon, Korea

Received: August 30, 2020

Revised: November 9, 2020

Accepted: November 24, 2020

\section{Correspondence to}

Sohyung Park

Department of Forensic Medicine,

National Forensic Service, 139

Jiyang-ro, Yangcheon-gu, Seoul

08036, Korea

Tel: +82-2-2600-4614

Fax: +82-2-2600-4828

E-mail: zzeva@korea.kr
The deceased was a 6-month-old male infant who was transferred to the hospital due to jaundice and vomiting. During admission, subdural hemorrhage and retinal hemorrhage were observed; additionally, a rib fracture was identified on the previous radiography images, which had eventually healed by the time of the follow-up study. This case was reported to the police, alleging child abuse, which led to a medicolegal dispute between the hospital and the deceased's parents. In addition, bleeding tendency and coagulopathy related to vitamin $\mathrm{K}$ deficiency were also found. The past history revealed that the deceased had diarrhea, jaundice, and experienced several episodes of acholic stools, and low levels of vitamin $D$ at birth. On postmortem examination, the brain already revealed liquefactive necrosis, but subdural hemorrhage was still observed. The optic nerves of both eyes revealed hemorrhage with focal retinal hemorrhage. No definite fracture was identified; however, osteopenia was observed in the long bones. The liver showed septal fibrosis and cholestasis which is nearly biliary cirrhosis, and mild dilation of the intrahepatic and extrahepatic bile ducts, suggesting a choledochal cyst. This case illustrates that subdural hemorrhage related to vitamin $\mathrm{K}$ deficiency could occur in an infant with a choledochal cyst. When subdural hemorrhage is encountered in a child with what is considered an intentional head injury, the possibility of spontaneous hemorrhage should be excluded.

Key Words: Subdural hematoma; Vitamin K deficiency; Choledochal cyst; Autopsy; Forensic sciences

\section{Introduction}

Subdural hemorrhage with retinal hemorrhage in infancy is a common finding that is usually observed in cases of abusive head trauma [1,2]. If a subdural hemorrhage is observed on postmortem examination or clinical image study in a pediatric case, the possibility of child abuse should be considered and the attending physician has a legal obligation to report a suspected child abuse case to the police or the National Center for the Right of the Child. However, given that subdural hemorrhage can also occur in metabolic disorders, clotting disorders, and in other medical conditions $[1,2]$, subdural hemorrhage encountered in a pediatric case should not be automatically considered as head trauma, and it is necessary that the case be meticulously and cautiously reviewed. We experienced a rare case in which the possibility of child abuse was initially suspected, but later considered as spontaneous subdural hemorrhage related to vitamin $\mathrm{K}$ deficiency in an infant with a choledochal cyst. 


\section{Case Report}

The deceased was a 6-month-old male baby. At birth, he was admitted to a hospital due to physiologic jaundice and transient tachypnea of the newborn. During admission, formula feeding was performed and diarrhea occurred, which was considered to be a milkprotein induced enterocolitis syndrome. Vitamin $\mathrm{K}$ was administered. The $25-\mathrm{OH}$-vit D level was low $(7.4 \mathrm{ng} /$ $\mathrm{mL}$ ). When he was 2 months old, he was admitted to a hospital due to urosepsis caused by extended spectrum beta-lactamase-negative Klebsiella pneumonia, and the left kidney revealed pelviectasia on the ultrasonogram. Vesicoureteral reflux grades were $3-4 / 4-5$ on voiding cystourethrography. After discharge, the deceased had to visit the outpatient clinic again due to recurrent fever. When he was 4 months old, he was admitted to a hospital because of acholic stool. Bile plug syndrome was considered on ultrasonography. Klebsiella species were identified in urine culture but he did not show any sign of fever. Alkaline phosphatase level was $1,555 \mathrm{U} / \mathrm{L}$ on blood test. When he was 5 months old, simple ureteroneocystostomy was performed on both sides. After the surgery, the acholic stool recurred. Probable multifocal bile plugs in the cystic duct of the gallbladder and common bile duct with mild dilatation of the intrahepatic duct and common bile duct, and focal dilation of the proximal cystic duct suggesting an underlying unusual choledochal cyst were observed on magnetic resonance imaging with magnetic resonance cholangiography (Fig. 1). He was admitted to another hospital for further evaluation because mild high forehead, mild upward slant of the eyes, epicanthal folds, low and broad nasal bridge, deformed ear lobe, and hepatosplenomegaly were observed on physical examination. Special tests for amino acids, very long chain fatty acid, and organic acids were performed, all of which came back normal. When the deceased was nearly 6 months old, he was admitted to a hospital because of irritability, jaundice aggravation, and two episodes of vomitin. His blood test revealed high total bilirubin (5.0 $\mathrm{mg} / \mathrm{dL}$ ), increased level of aspartate aminotransferase (104 U/L), low hemoglobin (8.6 g/dL), and increased number of white blood cells $(19,370 \mu \mathrm{L})$. On hospital day (HD) \#2, dyspnea and drowsy mental state were observed, and the general condition appeared worse with poor oral intake. Influenza B test was positive and blood tests revealed low hemoglobin $(6.2 \mathrm{~g} / \mathrm{dL})$ and an increased number of white blood cells $(31,690 \mu \mathrm{L})$. The parents reported that 2 days prior, the deceased's brother accidentally fell on the deceased's abdomen. On HD \#3, osteopenia and distal metaphyseal changes in both tibiae suspicious for rickets were observed on radiography images. Seizure-like symptoms, prolonged bleeding at intravenous access sites, shallow respiration, decreased oxygen saturation, and coagulopathy were observed. Subdural hemorrhage was identified on a brain computed tomography scan. On HD \#4, the mental status worsened to a coma, and the brain magnetic resonance imaging revealed multifocal multistate subdural hemorrhage, subarachnoid hemorrhage, and parenchymal hemorrhage suggesting axonal injury and, diffuse ischemic brain edema with transtentorial herniation. On ophthalmic examination, small dot hemorrhages were observed on the retina. Further evaluation was performed subsequently, and the possibility of bile plug syndrome followed by malabsorption of vitamins $A, D, E$, and $K$ was considered. The bleeding tendency with prolonged prothrombin

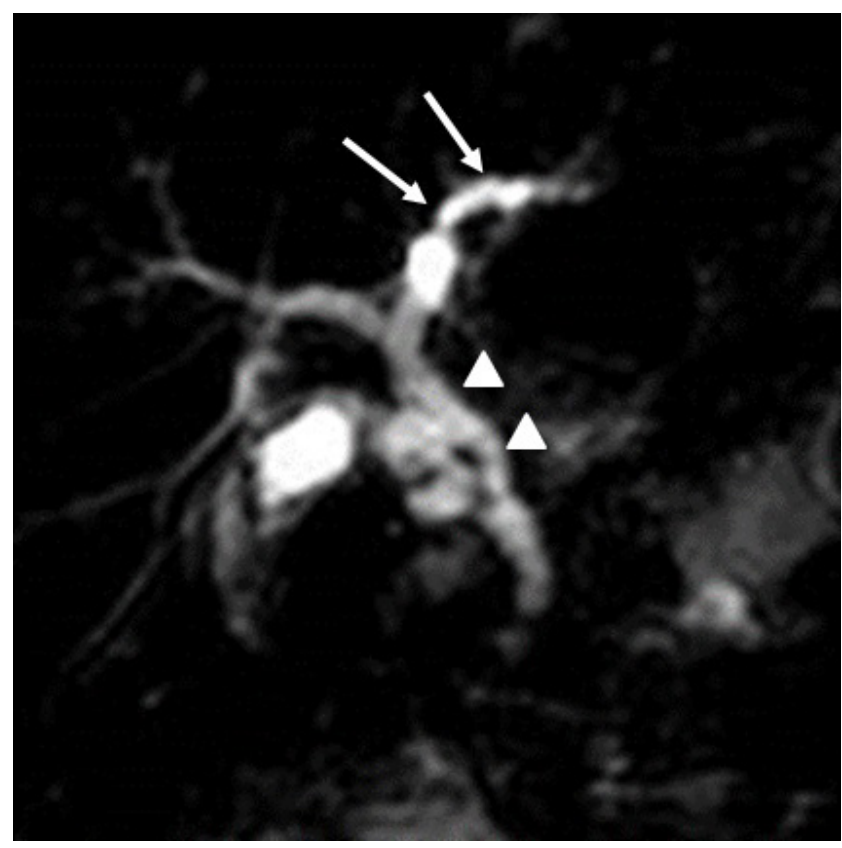

Fig. 1. Magnetic resonance imaging with magnetic resonance cholangiography reveals dilatation of the intrahepatic (arrows) and extrahepatic ducts (arrowheads) suggesting a choledochal cyst. 
time and partial thromboplastin time (PTT) and coagulation factor study revealed that the coagulopathy could be related to vitamin $\mathrm{K}$ deficiency. Ultimately the deceased's clinical course worsened, and he was expired on HD \#27. This case was reported to the police for suspected child abuse (or maltreatment) by the deceased's attending physician on $\mathrm{HD} \# 7$, based on the findings of subdural hemorrhage, retinal hemorrhages, and fracture of a rib identified on the review of the previous X-ray images in other hospitals. This case became a medicolegal dispute between the hospital and the parents and it was ultimately consulted for postmortem examination.

The deceased appeared well nourished but generalized edema was also observed. On external examination, mild upward slant of both eyes, low set ears, and umbilical hernia were observed. A focal hemorrhage $(2 \times 2 \mathrm{~cm})$ was identified on the posterior part of the parietal area. Needle puncture sites were observed on the left subclavian area and the anterior aspect of the right elbow. On internal examination, focal hemorrhage was identified around the posterior fontanelle and no fracture was identified on the skull. Thin subdural hemorrhage still remained and was observed on the dura (Fig. 2A). The brain parenchyma already revealed diffuse liquefactive necrosis, partly adhering to the dura. Perioptic hemorrhage was observed on the optic nerve of the left eye. Serous pleural fluid was identified in the thoracic cavity (approximately $100 \mathrm{~mL}$ and $70 \mathrm{~mL}$ in the left and right pleural cavities respectively), and serous ascites in the abdominal cavity (approximately $50 \mathrm{~mL}$ ). The common bile duct appeared be mildly dilated. The cut surface of the liver revealed septal fibrosis and mild dilatation of the intrahepatic and extrahepatic ducts (type IVa). The left kidney revealed atrophy and multifocal scars on the cortex, and the left pelvis and ureter revealed dilatation. Both vesicoureteral junctions of the urinary bladder appeared fibrotic and the distal part of both ureters were dilated. There was no fracture on the axial skeleton. Interstitial edema was observed in the soft tissue. On microscopic examination, the brain was necrotic and the dura revealed subdural hemorrhage with acute inflammation (Fig. 2B). The retina showed focal hemorrhage and perioptic hemorrhage was observed on the optic nerve (Fig. 2C). The liver showed septal fibrosis with ductular proliferation and cholestasis, which is nearly biliary cirrhosis (Fig. 2D). The kidney revealed focal interstitial fibrosis. The salivary glands revealed mononuclear inflammatory cells with cytopathic changes in some epithelia of the salivary ducts, suggestive of viral infection. The rib and femur had thin cortical bone and trabeculae, suggesting osteopenia (Fig. 2E). There was no definite abnormality on the growth plate. The results of the ancillary testing were unremarkable. The cause of death was assumed to be subdural hemorrhage but there were limitations related to the following changes as the clinical course worsened during admission. This study was approved by the Institutional Review Board (IRB approval number: 906-200114-HR-010-01).

\section{Discussion}

This case illustrates a rare case of spontaneous subdural hemorrhage related to vitamin $\mathrm{K}$ deficiency in an infant with a choledochal cyst. The clinical presentations such as repeated jaundice, diarrhea, acholic stool, and low levels of vitamin $D$ were assumed to be related to biliary dysfunction related to choledochal cyst and hepatic fibrosis, which was nearly biliary cirrhosis. The deceased also had some anatomical abnormalities, such as ambiguous facial morphologic features and vesicoureteral reflux followed by urosepsis and surgery (ureteroneocystostomy). Eventually the deceased's final clinical manifestations were assumed to be a symptomatic aggravation of a choledochal cyst, subdural hemorrhage with retinal hemorrhage, bleeding tendency, and osteopenia. On postmortem examination, subdural hemorrhage with perioptic hemorrhage was still observed, and mild dilatation of the intrahepatic and extrahepatic ducts suggesting a choledochal cyst and osteopenia were identified. All these clinical and postmortem findings supported that this case would be a spontaneous subdural hemorrhage due to biliary dysfunction from a choledochal cyst.

Trauma is the most common cause of subdural hemorrhage with retinal hemorrhage in infancy, regardless of whether it is an intentional or a nonintentional injury [1-3]. However, non-traumatic or 


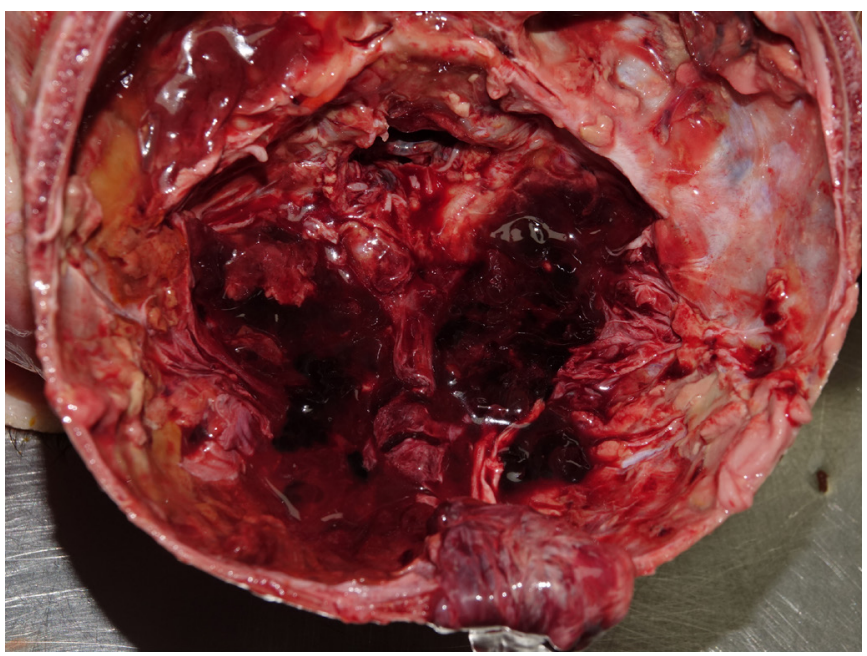

A

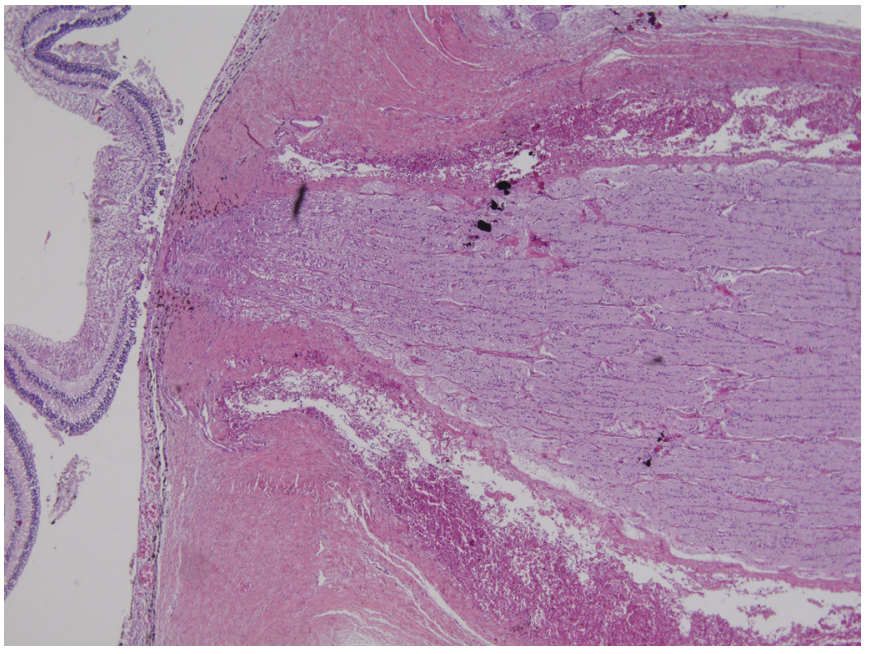

C

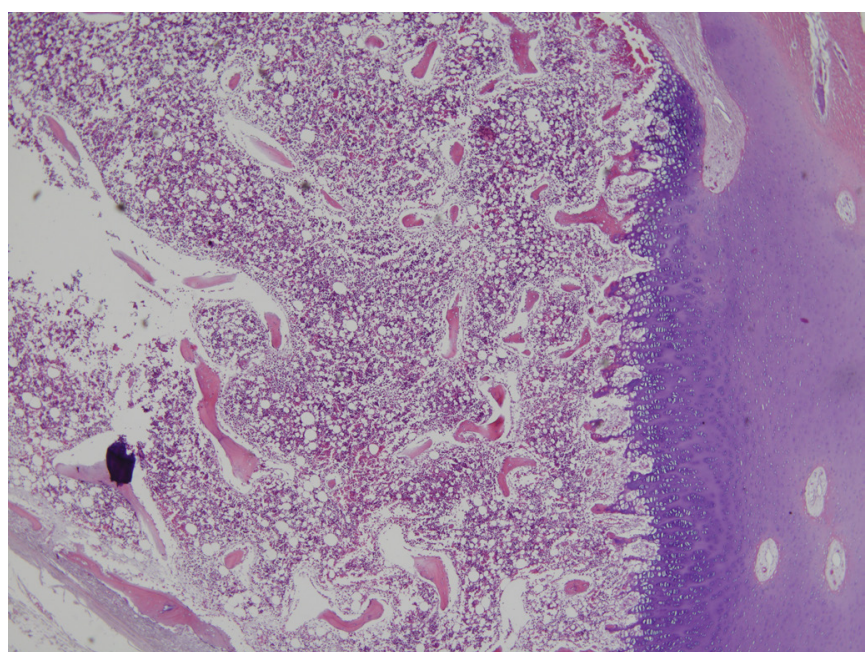

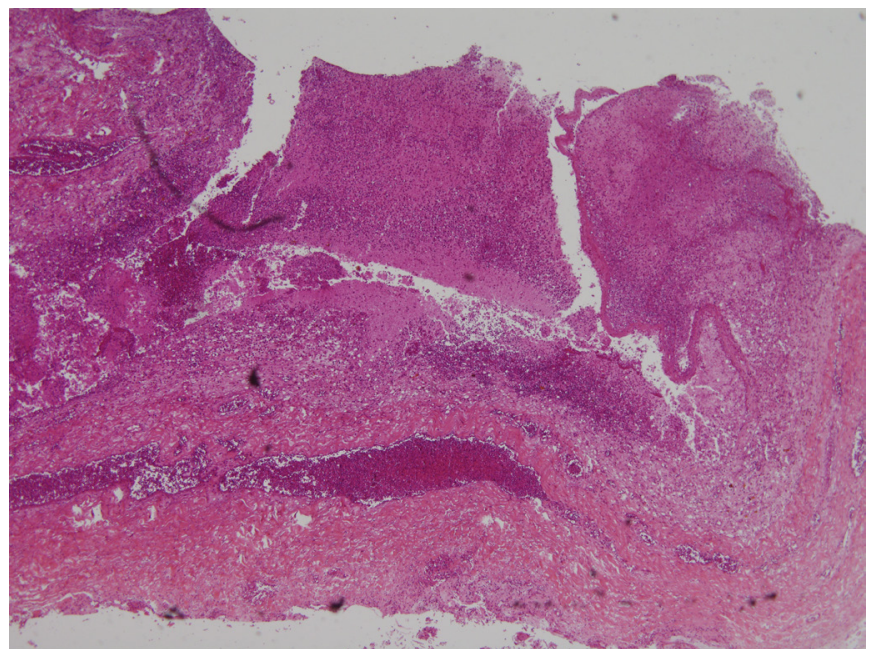

B

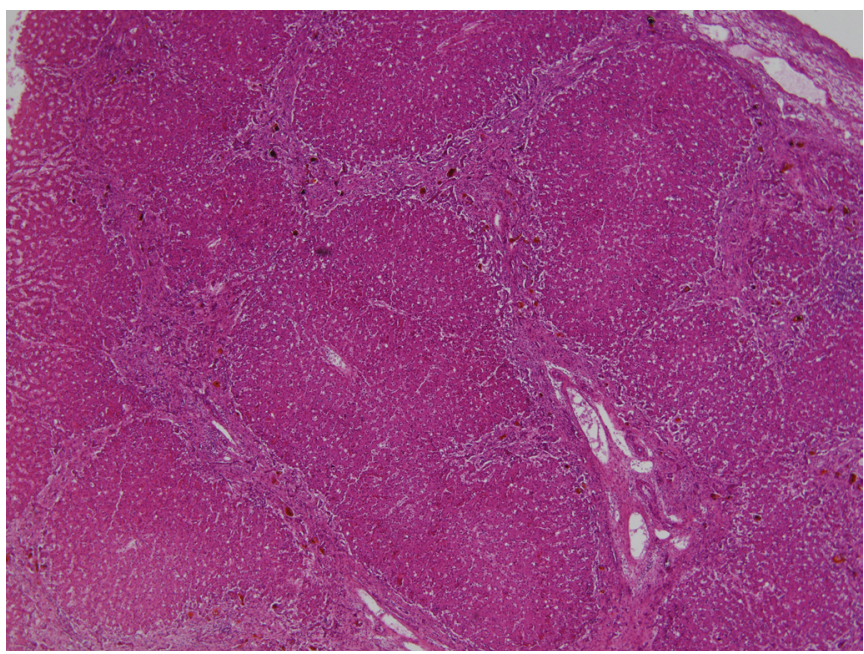

D

Fig. 2. Postmortem examination revealed several significant findings. Subdural hemorrhage is still observed on the dura of the skull base on internal examination (A) and on microscopic examination (B) $(H \& E$, $\times 40)$. (C) Perioptic hemorrhage is observed on the optic nerve ( $H \& E$, $\times 40$ ). (D) The liver shows hepatic fibrosis with cholestasis, close to biliary cirrhosis $(H \& E, \times 40)$. (E) Thin bony trabeculae are observed in the rib, suggesting osteopenia $(H \& E, \times 40)$.

E 
spontaneous subdural hemorrhage can occur during delivery, and in cranial malformation, meningitis, coagulation and hematologic disorders, metabolic disease and biochemical disorders (hypernatremia, dehydration), although it rarely occurs [1-4]. Retinal hemorrhage can also be observed in cases with sepsis, meningitis, bleeding disorders, vasculopathies, some genetic disorders, increased intracranial pressure, and so on [1]. In this case, there was a history of mild accidental trauma with the deceased's brother accidentally falling on the deceased's abdomen; however, it is unclear if there was a true trauma to the head. Focal hemorrhage was identified around the posterior fontanelle, but this would be more likely to be related to bleeding tendency rather than true trauma. The deceased's subdural hemorrhage with retinal hemorrhage was observed together with a bleeding tendency, which is assumed to be coagulopathy related to vitamin $\mathrm{K}$ deficiency. Therefore, this case was considered as spontaneous subdural hemorrhage related to vitamin K deficiency.

Coagulopathy or prolonged PTT can be a clinical manifestation of a choledochal cyst due to biliary obstruction $[5,6]$. There are a few reports of a bleeding tendency (gingival hemorrhages and nasal bleeding) related to vitamin $\mathrm{K}$ deficiency, subdural hemorrhage with subarachnoid hemorrhage and coagulopathy, or the presence of intracerebral hemorrhage with coagulopathy in infants with a choledochal cyst [79]. This case also ultimately had similar clinical manifestations; however, these were not the initial symptoms. Diarrhea, acholic stools, and jaundice which are known to be commonly observed in pediatric patients with choledochal cysts [10], seemed to have been initial symptoms that were presented several times.

In this case, the clinical review of previous radiography images identified a rib fracture, which had eventually healed, as observed later in the followup study. This finding was also considered suggesting a possibility of suspected child abuse, together with subdural hemorrhage and retinal hemorrhage. However, according to a study on pediatric patients with hepatobiliary disease, including one case of a choledochal cyst, rickets, or osteoporosis was observed in some pediatric patients, because the bile flow to the intestine would be deficient due to hepatobiliary diseases, which lead to vitamin D deficiency as well as vitamin $\mathrm{K}$ deficiency [11]. In this case, given that the deceased presented with low levels of vitamin $\mathrm{D}$ at birth and that osteopenia was observed, which increases susceptibility to fracture, the rib fracture would be assumed to be a pathologic fracture due to vitamin $\mathrm{D}$ deficiency related to a choledochal cyst.

In this case, the report to the police led to a medicolegal dispute between the hospital and the parents (the bereaved family). The clinical manifestations such as subdural hemorrhage, retinal hemorrhages, and previous rib fracture, a history of mild abdominal trauma by the deceased's brother, and no past history of bleeding tendency were suspected to be as a consequence of possible child abuse. Hence, this case was consulted for postmortem examination. Eventually, it was unlikely to be a case of child abuse, in the context of the postmortem findings, all clinical information from several hospitals, and the information from the police investigation report. However, the report by the attending physician was also reasonable as an initial response in this case, which led to the investigation and review of all information. Therefore this case implies that postmortem examination is critical in a dubious pediatric case.

In brief, we present a very rare case of spontaneous subdural hemorrhage related to vitamin $\mathrm{K}$ deficiency in an infant with a choledochal cyst. Subdural hemorrhage with retinal hemorrhage and rib fracture are usually observed in cases of child abuse, and in such cases, a careful and meticulous investigation reviewing the circumstances, family background, and home environment, would be necessary. However, as this case illustrates, and lest it should give unnecessary pain to the bereaved family, a postmortem examination with review of the entire clinical information and medical history and conditions is necessary. It is critical to establish medical evidence appropriately in a dubious pediatric case, and the possibility of spontaneous subdural hemorrhage should also be excluded.

ORCID: Sohyung Park: https://orcid.org/0000-00021987-7908; Jang Gyu Cha: https://orcid.org/0000-00023803-4850; Byung-Ha Choi: https://orcid.org/00000002-9401-1725 


\section{Conflicts of Interest}

No potential conflict of interest relevant to this article was reported.

\section{Acknowledgments}

This study was supported by the National Forensic Service Clinical Research Fund (NFS2020CLI09).

\section{References}

1. Collins KA, Byard RW. Forensic pathology of infancy and childhood. New York: Springer-Verlag; 2014. p 391-434.

2. Squier W, Mack J. The neuropathology of infant subdural haemorrhage. Forensic Sci Int 2009;187:6-13.

3. Kemp AM. Investigating subdural haemorrhage in infants. Arch Dis Child 2002;86:98-102.

4. Gopakumar H, Sivji R, Rajiv PK. Vitamin K deficiency bleeding presenting as impending brain herniation. J Pediatr Neurosci 2010;5:55-8.
5. Diao M, Li L, Cheng W. Coagulopathy in a subtype of choledochal cyst and management strategy. World J Gastroenterol 2014;20:10606-12.

6. Badebarin D, Aslanabadi S, Teimouri-Dereshki A, et al. Different clinical presentations of choledochal cyst among infants and older children: a 10-year retrospective study. Medicine (Baltimore) 2017;96:e6679.

7. Ono S, Tokiwa K, Aoi S, et al. A bleeding tendency as the first symptom of a choledochal cyst. Pediatr Surg Int 2000;16:111-2.

8. Chen TY, Wang HK, Yeh ML, et al. Subdural hemorrhage as a first symptom in an infant with a choledochal cyst: case report. J Neurosurg Pediatr 2012;9:414-6.

9. Ali SW, Manzoor N, Ashraf MS, et al. Intra-cerebral hemorrhage associated with choledochal cyst in an infant.J Pediatr Surg Case Rep 2020;58:101373.

10. Soares KC, Goldstein SD, Ghaseb MA, et al. Pediatric choledochal cysts: diagnosis and current management. Pediatr Surg Int 2017;33:637-50.

11. Kobayashi A, Kawai S, Utsunomiya T, et al. Bone disease in infants and children with hepatobiliary disease. Arch Dis Child 1974;49:641-6. 